\title{
An Application of Intuitionistic Fuzzy in Routing Networks
}

\author{
Ashit Kumar Dutta \\ Dept. of Computer Science \\ Alquwaya college of Science, Shaqra University \\ Kingdom of Saudi Arabia
}

\author{
Abdul Rahaman Wahab Sait \\ Dept. of Computer Science \\ Alquwaya Community College, Shaqra University \\ Kingdom of Saudi Arabia
}

\begin{abstract}
Routing is an important functional aspect of networks to transport packets from source to destination. A router sets up optimized paths among the different nodes in the network. In this paper the authors proposed a new type of routing algorithm which includes exchange of routing information for small amount of time and then halts for few hours but this information is used by each router to make its own routing decisions based on intuitionistic fuzzy logic during these hours.
\end{abstract}

Keywords- component; Intuitionistic fuzzy set; fuzzy set; intuitionistic fuzzy routing; fuzzy routing; GV; IFV; MEO; IMEV; IFLED.

\section{INTRODUCTION}

The growing usage of computer networks is requiring improvements in network technologies and management techniques so users will receive high quality service. As more individuals transmit data through a computer network, the quality of service received by the users begins to degrade. A more effective method for routing data through a computer network can assist with the new problems being encountered with today's growing networks.

Broadband ISDN (B-ISDN) was envisioned as a provider of higher bit rates to the user than N-ISDN. One of the key design objectives of B-ISDN is that the provision of a wide range of services to a broadband variety of users utilizing a limited set of connection types and multi-purpose user-network interfaces. The two prominent enabling technologies for the deployment of B-ISDN are fiber optics and ATM network architecture. ATM refers to switching and multiplexing techniques. As ATM has to support wide range services whose requirements vary over a wide range, the transport of cells must be at high speed. This calls for minimizing the processing time at the intermediate devices like router and the efficient methods for traffic management.

Increasing traffic loads will naturally lead to network delays, which will lead to other problems as well. These network delays can easily cause dropped sessions or lost data, not to mention dissatisfied users. It is impossible to stem this increasing traffic load. However, optimal routing of messages within a network can mitigate some of the difficulties of heavy traffic. Therefore, a more efficient method of routing needs to be developed to combat network delays.

Routing is an important functional aspect of networks to transport packets in general (or cells in ATM networks) from source to destination. A router sets up optimized paths among the different nodes in the network. An optimized path is that one which gives low mean packet delay and high network throughput. Many routing algorithms exist in the literature. All these can be broadly classified into static and dynamic algorithms. Dynamic routing algorithms make decision regarding the optimized paths independently of other routers based on the information exchanged among the adjacent routers. This exchange of routing information is carried out periodically increasing the traffic on the network.

In [5], we proposed a new type of routing algorithm which includes exchange of routing information for small amount of time and then halts for few hours but this information is used by each router to make its own routing decisions based on intuitionistic fuzzy logic during these hours. The new routing may be called "Intuitionistic Fuzzy Routing". The "fuzzy routing" is a special case of intuitionistic fuzzy routing as usual.

Dynamic routing algorithms exchange routing information periodically among the adjacent routers. This is called periodic updates. This period typically ranges from few tens of milliseconds to 1 or 2 minutes. If the updates are too frequent, congestion may occur. On the other hand, if updates are too infrequent, routing may not be efficient. Hence these dynamic algorithms add extra traffic due to the exchange of routing information among the routers to the network. Traffic due to ever increasing demand of new services is also growing. This is making the traffic (due to routing and user information) management issue a complex one and hence it is becoming a major field of research in present days networks like ATM. Hence the authors are making an attempt to reduce the traffic due to routers by embedding intuitionistic fuzzy intelligence into existing adaptive routers. These intuitionistic fuzzy routers are expected to increase the speed of routing as compared to conventional adaptive routers as frequent exchange of routing information is not required.

\section{EXISTING Routing AND EXISTING FuZZY Routing}

In today's Internet world, information, split into small blocks called packets or cells, is moved across some kind of networks and terminates at distance point. In this process, a data packet passes through a route or path identified by routers. Hence routing is an important issue to communicate among the users on different networks. The selection of optimized path between sender and receiver is a major design area of network 
layer of ISO's OSI reference model [1]. Many algorithms for routing are available in the literatures which fall in one of two major groups: non-adaptive and adaptive. Non-adaptive (also called static) algorithms do not base their routing decisions on measurements or estimates of the current traffic and topology.

Adaptive (also called dynamic) algorithms, in contrast, change their routing decisions to reflect changes in the topology and traffic. These adaptive algorithms decide the routing path based on the information they get from other routers. The various adaptive algorithms, available in the literature, differ in the way they get information (locally, from adjacent routers or from all routers), when they change the routes (e.g. every $\mathrm{t} \mathrm{msec}$, when the load changes, or when the topology changes), and what parameter is used for optimization (e.g. distance, number of hops, or estimated transit time, reliability, bandwidth, load etc). These adaptive routing algorithms are much applicable in the present scenario of changing networks both in size and service requirements.

Dynamic routing algorithms exchange routing information periodically among the adjacent routers. This is called periodic updates. This period typically ranges from few tens of milliseconds to 1 or 2 minutes. If the updates are too frequent, congestion may occur.

On the other hand, if updates are too infrequent, routing may not be efficient. Hence these dynamic algorithms add extra traffic due to the exchange of routing information among the routers to the network. Traffic due to ever increasing demand of new services is also growing. This is making the traffic (due to routing and user information) management issue a complex one and hence it is becoming a major field of research in present days networks like ATM.

Hence the authors are making an attempt to reduce the traffic due to routers by embedding intuitionistic fuzzy intelligence into existing adaptive routers. These intuitionistic fuzzy routers are expected to increase the speed of routing as compared to conventional adaptive routers as frequent exchange of routing information is not required.

Distance Vector Routing algorithm, originally proposed by Bellman[2], Ford and Folkerson[4], is considered as conventional dynamic routing algorithm as it is being employed widely in today's networks like Routing Information Protocol (RIP) for IP, Cisco's Internet gateway Routing Protocol (IGRP), AppleTalk's Routing Table Maintenance Protocol (RTMP) etc.

In [7], the authors proposed a rule based fuzzy logic to find out the optimum path considering the crisp values of hop count metric. Further, testing and simulation study of the approach is carried out with different traffic loads. It is concluded that the approach decreases the processing overhead and provides a fair distribution of network traffic as compared to other traditional

\begin{tabular}{|c|c|c|c|c|c|}
\hline 1 & 2 & 3 & 4 & $\ldots \ldots$ & $\mathrm{k}$ \\
\hline${ }_{1 j} \quad \begin{array}{l}\mathrm{V} \\
\end{array}$ & ${ }_{2 j} \quad \mathrm{~V}$ & ${ }_{3 j} \quad \mathrm{~V}$ & ${ }_{4 j} \quad \begin{array}{l}\mathrm{V} \\
\end{array}$ & $\cdots \cdots$ & $\mathrm{kj}^{\mathrm{V}}$ \\
\hline
\end{tabular}

routing techniques.

In [6], intelligent fuzzy approach is proposed for routing the tagged cells. Simulation results showed an improvement in network utilization. The fuzzy routing reported by the authors $[8,9]$ in queuing systems and computer network routing are different from the fuzzy routing proposed by us in this work.

In Distance Vector Routing, each router maintains a routing table containing one entry for each destination in the network. This entry tells the preferred outgoing line to use for that destination. The router knows the "distance" (number of hops, queue length or delay) to each of its neighbors. For example, consider that delay is used as a metric and assume that the router knows the delay to each of its neighbors. Once every $t$ msec, each router sends to each neighbor a list of its estimated delays to each destination. It also receives a similar list from each neighbor.

Based on this information, a router can find out which estimate seems the best and updates its routing table. This routing table will be used by the router to route the packets for next $\mathrm{T}$ msec $(\mathrm{T}>\mathrm{t})$, after which routing information will be exchanged again and this procedure is repeated. Thus for every $\mathrm{t}$ msec, routing information will be exchanged among the adjacent routers which leads to increased traffic on the network. But the advantage of the algorithm is that it updates routing information dynamically for every fixed time interval. In this paper, we propose a method to reduce the traffic due to exchange of routing information in Distance Vector Routing algorithm retaining its advantage mentioned above.

Our method is mainly based on fuzzy mathematics to deal with the uncertainties in traffic handling. We introduce some new terminologies in section-3 and then in section-4 we explain our method of fuzzy routing. The work reported here covers the theoretical aspects of fuzzy routing whereas implementation and simulations will be our future research plan.

\section{InTUITIONISTIC MOST EXPECTED VECTOR (IMEV)}

In this section, we present some preliminaries on Intuitionistic Most Expected Vector. This notion of IMEV is used by us in [5] on intuitionistic fuzzy routing technique.

\section{A. Generated Vector $(G V)$}

Consider $\mathrm{k}$ number of $\mathrm{n}$-dimensional vectors $\mathrm{V} 1, \mathrm{~V} 2, \ldots \ldots$ , $\mathrm{Vk}$

$$
V_{i}=\left[\begin{array}{c}
\nu_{i 1} \\
v_{i 2} \\
\cdot \\
- \\
v_{i n}
\end{array}\right], i=1,2,3, \ldots, k
$$

where

For each $\mathrm{j}(\mathrm{j}=1,2, \ldots, \mathrm{n})$, we will do here $\mathrm{n}$ number of extrapolations by using Newton's Backward Interpolation formula, for the $\mathrm{n}$ tables given by 
to

calculate $v k+1, j, \quad j=1,2,3, \ldots \ldots ., n$.

Thus a new vector is generated, which is

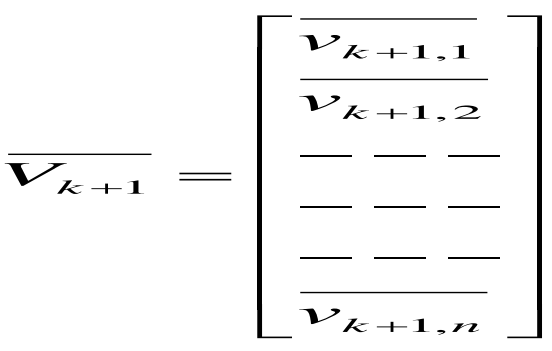

$$
\left\{\begin{aligned}
\theta\left(x_{i}\right) & =\frac{\mu_{A}\left(x_{i}\right)}{v_{A}\left(x_{i}\right)}, & & \text { if } v_{A}\left(x_{i}\right) \geq \pi_{A}\left(x_{i}\right) \\
& =\frac{\mu_{A}\left(x_{i}\right)}{\pi_{A}\left(x_{i}\right)}, & & \text { otherwise }
\end{aligned}\right.
$$

D. Intuitionistic Most Expected Vector (IMEV) where

Suppose that IFV $(\mathrm{V} 1, \mathrm{~V} 2, \ldots ., \mathrm{Vk})=\tilde{V}_{k+1}$,

$$
V_{k+1}=\left[\begin{array}{c}
\tilde{v}_{k+1,1} \\
\tilde{v}_{k+1,2} \\
--- \\
---- \\
---- \\
\tilde{v}_{k+1, n}
\end{array}\right]
$$

The Most Expected Vector (IMEV) of $\tilde{V}_{k+1}$ is the vector

$$
V_{k+1}=\left[\begin{array}{l}
\operatorname{MEO}\left(\widetilde{v}_{k+1,1}\right) \\
\operatorname{MEO}\left(\widetilde{v}_{k+1,2}\right) \\
------ \\
------ \\
------ \\
\operatorname{MEO}\left(\widetilde{v}_{k+1, n}\right)
\end{array}\right]=\left[\begin{array}{c}
v_{k+1,1} \\
v_{k+1,1} \\
--- \\
--- \\
--- \\
v_{k+1, n}
\end{array}\right], \quad \text { (say) }
$$

which we denote by $\operatorname{IMEV}(\mathrm{V} 1, \mathrm{~V} 2, \ldots ., \mathrm{Vk})$.

\section{InTUITIONISTIC FUZZY ROUTING [5]}

In [5], we presented our proposal for intuitionistic fuzzy routing. This is an application of Atanassov's[2,3] theory of IFS in routing. Suppose that the size of the network is $r$ (the number of routers or nodes). We assume that all the routers are active and the metric is measured by delay.

Consider two choice parameters $\mathrm{n}$ and $\mathrm{T}$ (to be precisely understood later on).

Method:

Once every t msec, each router sends to each of its neighbor a "list of estimated delay" (LED) to reach the every router of the network. On sending n number of such lists, it halts for $\mathrm{T}$ hours.

After T hours, the router again sends a fresh set of LED and so on. Clearly each LED is a r-dimensional vector. Our work in this paper presents a method for generating a new type of LED which we call here Intuitionistic Fuzzy LED (IFLED). 
For the sake of presentation of our method, let us consider the subnet as shown in Fig.1

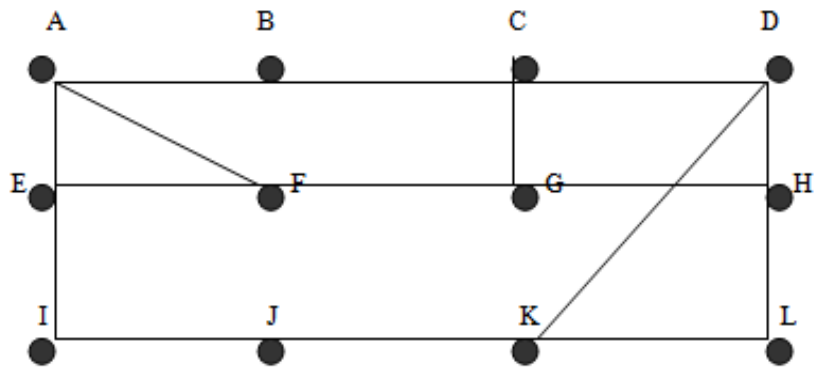

Figure 1: A Subnet

With no loss of generality consider any router, say F. It has three neighbors A, E and G. Therefore it can receive LED from these three neighbors only. Suppose that at some instant $\tau$, and then at the regular instants $(\tau+\mathrm{t}),(\tau+2 \mathrm{t}), \ldots .,(\tau+(n-1) t)$, the router $F$ receives the following sets of three LEDs from $A$, $\mathrm{E}$, and $\mathrm{G}$ respectively.

$L E D$ information received by the router $F$

\begin{tabular}{|c|c|c|c|}
\hline $\begin{array}{c}\text { At } \\
\text { the } \\
\text { instant }\end{array}$ & $\begin{array}{c}\text { LED } \\
\text { received } \\
\text { from A }\end{array}$ & $\begin{array}{c}\text { LED } \\
\text { received } \\
\text { from E }\end{array}$ & $\begin{array}{c}\text { LED } \\
\text { received } \\
\text { from G }\end{array}$ \\
\hline$\tau$ & $V_{0}^{A}$ & $V_{0}^{E}$ & $V_{0}^{G}$ \\
\hline$\tau+\mathrm{t}$ & $V_{1}^{A}$ & $V_{1}^{E}$ & $V_{1}^{G}$ \\
\hline $\begin{array}{c}\tau+ \\
2 \mathrm{t}\end{array}$ & $V_{2}^{A}$ & $V_{2}^{E}$ & $V_{2}^{G}$ \\
\hline $\begin{array}{c}\tau+ \\
(\mathrm{n}-1) \mathrm{t}\end{array}$ & $V_{n-1}^{A}$ & $\ldots$ & $\cdots$ \\
\hline
\end{tabular}

Each entry vector in the above table is r-dimensional (in this example $r=12$ ).

$$
\text { Forexample(hypothetic al) } V_{3}^{C}=\left[\begin{array}{l}
x_{1} \\
x_{2} \\
\cdot \\
\cdot \\
\cdot \\
x_{r}
\end{array}\right],
$$

then it means that the optimal delay from $\mathrm{C}$ to the router $\mathrm{R} 1$ is $\mathrm{x} 1$, the optimal delay from $\mathrm{C}$ to the router $\mathrm{R} 2$ is $\mathrm{x} 2$, etc.

Now let us find the following three vectors:

$$
\operatorname{IMEV}\left(V_{0}^{A}, V_{1}^{A}, \ldots \ldots, V_{n-1}^{A}\right)=V_{n}^{A}
$$
(2) $\operatorname{IMEV}\left(V_{\mathrm{O}}^{E}, V_{1}^{E}\right.$ $\left.V_{n-1}^{E}\right)=V_{n}^{E}$
, (say)
(3) $\operatorname{IMEV}\left(V_{\mathrm{o}}^{G}, V_{1}^{G}, \ldots \ldots, V_{n-1}^{G}\right)=V_{n}^{G}$, (say).

The collection of these three vectors is called Intuitionistic Fuzzy LED ( IFLED). During the computation of these three vectors, the router $F$ will be functioning according to the previous IFLED. The new IFLED now will remain valid to the router $F$ for $T$ hours next, until the next IFLED is computed. With the help of this IFLED, routing will be done by F. Once an IFLED is computed, the previous IFLED gets replaced.

\section{CONCLUSION}

The work done in [5] is an application of Atanassov's Intuitionistic fuzzy set theory [2, 3] in network. We have proposed a new method of routing called intuitionistic fuzzy routing. We have considered Distance Vector Routing with intuitionistic fuzzy tools.

Consider the traffic situation on the roads of a city. During a time interval of $\mathrm{T}$ hours (say 30 minutes), the mean traffic on a road could be assumed to be almost equal. During the next 30 minutes, again it is almost equal with another mean value of traffic. This is the philosophy we have used in [5]. The intuitionistic fuzziness in the value of almost equal mean of traffic is to be dealt with proper membership function and non-membership function.

The method proposed is both dynamic as well as static. The method does not need continuous resource management traffic, but it works on updated information (updated every $\mathrm{T}$ hours). Thus a gain in the reduction of resource management traffic has been made possible. It is claimed that the method will improve the overall performance of the network.

The disadvantage of the method is that in case a catastrophic traffic of packets arises in any path, the method may not yield better performance.

The work for the construction of membership and nonmembership functions for generating IFLED in intuitionistic fuzzy routing needs a rigorous study and survey, which is under our next research plan.

If fortunately, all the indeterministic functions are null functions (i.e. if there is no hesitation), intuitionistic fuzziness reduces to fuzziness and then the intuitionistic fuzzy routing will be reduced to 'fuzzy routing' as a special case[5]. The fuzzy routing reported by the authors $[8,9,10]$ in queuing systems and computer network routing are different from the fuzzy routing proposed by us.

\section{REFERENCES}

[1] A. S. Tanenbaum., Computer Networks, Prentice-Hall, Englewood Cliffs, NJ, 1988.

[2] Atanassov, K., Intuitionistic Fuzzy Sets, Fuzzy Sets and Systems. 20(1986) 87-96.

[3] Atanassov, K., Intuitionistic Fuzzy Sets : Theory and Applications, Physica-Verlag, Heidelberg (1999). 
[4] Bellman,R.E., Dynamic Programming, Princeton, NJ; Princeton University Press, 1957

[5] D. Driankov, H. Hellendoorn and M. Reinfrank, An Introduction to Fuzzy Control. Springer-Verlag, Berlin, New York, 1993.

[6] Ford,L.R. and Fulkerson,D.R., Flows in Networks, Princeton,NJ; Princeton University Press, 1962.

[7] Ka-Wing Wong, "Fuzzy routing control of service request messages in an individual computing environment", Proc. of the 1995 ACMSymposium on Applied Computing, Nashville (USA), 1995, pp 548-551.

[8] Runtong Zhang and Yannis A. Phillis, "Fuzzy Routing of Queueing Systems with Heterogeneous Servers", Proc. of IEEE Intl. Conference on Robotics and Automation Albuquerque, New Mexico, April 1997, pp. 2340-2345.

[9] Runtong Zhang and Yannis A. Phillis, "Fuzzy Service Rate Control of Queueing Systems", Proc. 1996 IEEE Intl. Conf. On Systems, Man and
Cybernetics on Information, Intelligence and Systems (IEEE/SMC'96), October 14-17, 1996, Beijing, China.

[10] Sridhar Pithani and Adarshpal S. Sethi, "A fuzzy Set Delay Representation for Computer Network Routing Algorithms" Proc. Second Intl. Symposium on Uncertainty Modeling and Analysis, College Park, MD (April 1993), pp. 286-293.

[11] Zadeh, L. A., Fuzzy Sets, Information and Control. 8(1965) 338-353.

[12] Gasim Alandjani and Eric. E.Johnson,"Fuzzy routing in Ad hoc networks", IEEE 2003.

[13] Barolli L, Koyama A, Yamada T, and Yokoyama S, "An Intelligent fuzzy routing scheme for improving ATM Network Performace using Violation tagging function", IEEE Proceedings on Database and expert system application, Iss: 2000, pp. 5 -9.

[14] Dr.Shuchitha Upadhayay and Mini Sharma, "Reinforcement of a new fuzzy mixed metric approach through fuzzy routing Algorithms", IJCNS, Vol.8,No.2, Feb 2008, pp $271-276$. 